\title{
Improving the management of patients with acute red eyes in a large London Emergency Department
}

\author{
Aamir Saifuddin, Rachel Brookes \\ King's College Hospital, London
}

\begin{abstract}
Anecdotal evidence suggested that the management of patients with eye complaints in the Emergency Department (ED) at King's College Hospital, London was suboptimal. Acute ophthalmology is often poorly covered at undergraduate level in the United Kingdom which can affect patient safety. Furthermore, it was notoriously difficult to obtain specialist advice within working hours. Contact lens (CL) wearers are prone to Pseudomonas conjunctivitis which requires certain antibiotics and mismanagement of this has led to sight-threatening consequences locally. Junior doctor surveys suggested under-confidence in managing eye problems and initial audit showed that eye assessments are frequently incomplete during ED clerkings. For example, CL status and visual acuity were documented in $63 \%$ and $77 \%$ of cases respectively; however, these were increased when a dedicated pro forma was used. To address these multiple issues, a new 'eye examination pro forma' was created, along with integrated clinical guidelines based on local expert practice. This would prompt staff to elicit key information to help guide management. A new referral pathway was also introduced to facilitate access to ophthalmology services. On re-auditing seven months later, the new pro forma was completed for only $28 \%$ of patients, though this was associated with a higher rate of documentation of all parameters. The referral pathways worked efficiently and patients with red flag features were identified and managed more appropriately than before. We learnt that it is important that someone personally drives the innovations from the outset, otherwise prolonged change is difficult. Secondly, junior doctors may not be proficient with slit lamp use, for instance, so targeted teaching is required, not simply new pathways. Full engagement with the pro forma and effective patient management should then improve simultaneously. Specific teaching is now being implemented and permanent staff have been recruited to oversee the project. We plan to re-audit in November 2014.
\end{abstract}

\section{Problem}

As with most Emergency Departments (EDs), King's College Hospital in London in the United Kingdom (UK) deals with a large range of acute medical problems every day and relies upon a diverse cohort of clinicians to manage these. Amongst this plethora of patients, personal experience and anecdotal evidence suggested that optimising the care of those with acute eye problems was difficult for several reasons. Firstly, clinical ophthalmology, especially in the emergency setting, is often poorly covered at medical school in the UK. Secondly, most juniors may see very few such cases during an entire four-month rotation, so there is not enough early exposure to generate a working knowledge of the field. Thirdly, optimal management of these patients includes skills such as slit lamp use or interpreting fluorescein stain results, which need to be learnt either by seeking senior support when seeing a patient or from separate teaching sessions. However, given the shift patterns and time pressures in the ED, each of these is difficult and achieving competence in these skills is rare; juniors, therefore, often elect not to use them. Fourthly, the on-call ophthalmology registrar was normally in clinic so it became notoriously difficult to gain specialist advice during working hours, which is when $48 \%$ of patients with "eye problems" present (data from previous local audit). If they were not available, guidance needed to be sought from eye clinic nurses, who often had limited ophthalmology experience, and there was also little clinical guidance on the intranet, in great contrast to most other common ED complaints.
350,444 ED presentations for "ophthalmological conditions", which comprised $3.0 \%$ all attendances; the figure was similar (3.1\%) the previous year. [1] This was the tenth most common "ED diagnosis" out of 38 categories; "cardiac condition" was ninth in this list at $3.6 \%$. This demonstrates the relative frequency of eye problems and reiterates the fact that substandard care is unacceptable, as it would be for mismanagement of acute heart complaints. Local audit found that there were 649 admissions triaged as "eye problems" in the three months from July to October 2012, which extrapolates to $1.8 \%$ of overall ED admissions.[2] This disparity in local and national figures is likely because King's College Hospital is not a tertiary referral centre for ophthalmology, whilst it is for various other specialties including trauma, stroke and neurosurgery, which will skew the percentage figures.

The majority of patients are adults who present for the first time to the ED, with very few (2\%) referred by their General Practitioner or High Street optician; such patients might bypass ED and attend the eye clinic directly or might be sent to a specialist centre. Overall, $48 \%$ present in-hours (0900 to 1700 ) and $73 \%$ are seen on weekdays. There is an average of 7.31 patients with "eye problems" each day during the week and 6.44 over the weekend. [2] There are, therefore, enough attendances that clinicians need to know how to manage them effectively, but also sufficiently few that junior doctors may not gather much specific experience, especially if the patients are seen by Emergency Nurse Practitioners (ENPs) during the day.

New systems therefore needed to be implemented to address these 
problems and ensure that patient care is not affected.

\section{Background}

Following advice from senior ED and ophthalmology staff, we decided to focus on patients presenting acutely with red eye(s). They have large scope for mismanagement as they are often assessed relatively quickly by junior doctors who, as detailed above, may not have the necessary experience or support to optimise their care. This especially applies to contact lens $(\mathrm{CL})$ wearers who are at risk of sight-threatening microbial keratitis by the Gram-negative bacterium, Pseudomonas aeruginosa. This damages the cornea directly by releasing proteases and indirectly by over-activating the host immune system. [3] These patients therefore benefit from different antibiotics compared to non-CL wearers and need closer follow-up, as advised by local ophthalmologists and the American Optometry Association. [4] Nonspecialists may be unaware of these important facts and this could lead to disastrous consequences for the patient. The Trust has also been heavily implicated with large financial costs in the past, including legal settlements and ongoing treatment such as corneal grafting and outpatient care. Similarly, there may be far-reaching reputational damage.

In order to improve and standardise the management of these patients, an 'eye clerking pro forma' had been devised by one of the senior ENPs two years previously. However, it was concerned with identifying minute clinical details rather than helping the nonspecialist ED clinician to come to a diagnosis or to effectively triage the patients who needed an urgent specialist opinion. It was convoluted and completed only $13 \%$ of the time and not based on specialist advice or guidelines.

Interestingly, the National Institute for Health and Clinical Excellence in the UK (NICE) does not specify that CL wearers should be given different antibiotics, though Pseudomonas is not specifically listed as a common bacterial eye infection.[5] In fact, a systematic review of the literature pertaining to acute infective conjunctivitis management in Primary Care, which includes placebocontrolled trials, suggests that most occurrences of bacterial conjunctivitis are self-limiting with rare serious complications. Whilst topical antibiotics can improve recovery, antimicrobial prescription is often not necessary.[6] Nevertheless, within our ED setting, with the reduced ability for close follow-up compared to Primary Care, antibiotics are prescribed routinely.

A search in BMJ Quality Improvement Reports for "contact lens" or "conjunctivitis" revealed no results, suggesting that this is a poorly researched area. Similarly, there were no corresponding quality improvement projects found when searched via PubMed or Google.

Our aims were two-fold. Firstly, we wanted to improve the safety of patients presenting to the ED with "red eye(s)", especially $\mathrm{CL}$ wearers, by improving the quality of the medical assessment they receive, along with their initial management and safety net or followup plans. Secondly, we hoped to improve patient flow within the ED by creating easier access to ophthalmology specialists throughout the day and by devising guidance about when to refer. Time would then be saved in clinical decision-making and not wasted by seeking senior ED advice before the referral is made.

\section{Baseline measurement}

Surveys were carried out among local Foundation Year 2 doctors (FY2s) $(n=21)$ (see attached files). When asked to score their level of confidence in dealing independently with common presentations, "red eye" and "visual disturbance" averaged 2.3 and 2.2 out of 5 respectively - the lowest scores - in contrast to others complaints such as diarrhoea and vomiting (4.0), abdominal pain (3.7), and painful limb (3.3). Furthermore, $65 \%$ of participants stated that ophthalmology teaching at medical school had not prepared them well for their ED placement and $60 \%$ felt that more teaching as junior doctors would help.

In order to gauge any specific areas of substandard management, we audited a consecutive series of one hundred casualty cards for all patients over 18 years-old with an ultimate diagnosis of "conjunctivitis" who presented from February to April 2013, as recorded on the electronic Symphony(TM) system by all clinicians when their patient is to be discharged from the department. Any incidences where the diagnosis was not felt to be bacterial conjunctivitis (i.e. where antibiotics would not be indicated), either by the ED clinician or on our retrospective analysis of the full case notes, were not included in our data. Note was made regarding the documentation of $\mathrm{CL}$ status, formal acuity testing and the appropriate use of pinhole correction, fluorescein staining, slit lamp use, the documentation of a safety net or follow-up, and the presence of 'red flag' clinical features and whether or not these patients were referred to the ophthalmologist on-call. If any aspect was not documented, we inferred that it had not been carried out.

We found that, in $37 \%$ of cases, CL status was not documented and in $23 \%$ of patients, formal Snellen chart acuity testing was not performed; in a further $10 \%$, this was not documented in a standard notation so it would be difficult to interpret its meaning medicolegally. Pinhole testing is deemed appropriate by the ophthalmology team when acuity is less than or equal to $6 / 9$. When acuity was found to be this low, a pinhole was employed only $28 \%$ of the time. Fluorescein staining and the slit lamp were used in only $42 \%$ of cases. There was no safety net for $33 \%$ of patients. Worryingly, in patients with red flag features, only $53 \%$ were referred acutely to the specialists. This included clinical scenarios such as 'red eye with vomiting', ulceration on slit lamp examination and chemical injury. However, most concerning was that five patients were identified as being contact lens wearers with red eyes but were discharged with the incorrect antibiotic, in some cases by senior staff. Two of these had no follow-up arranged. Interestingly, when the pro forma was used, $94 \%$ of patients had contact lens status documented; when the pro forma not used, this figure was $58 \%$.

See supplementary file: ds3405.pdf - "Pre-intervention data and old pro forma"

\section{Design}


Clerking pro forma and clinical guidance

Our data above shows that $\mathrm{CL}$ status documentation was better when a pro forma was used, suggesting that a revised version might be an appropriate intervention. Unfortunately, there were no official guidelines for what level of detail should be included in an ED eye examination from any organisations, including the respective Royal Colleges. We therefore developed a new "eye examination pro forma" ourselves, in close conjunction with senior ophthalmology and emergency medicine colleagues. It was specifically designed so that it would prompt clinicians to examine the domains that the specialists would need for triaging patients over the phone. This would help to optimise management and improve efficiency within the ED.

We also sought to improve the ophthalmology guidelines on the intranet. The new guidelines would need to streamline the referral pathway, help ED staff to know which patients to refer and provide acute clinical advice. We decided that the most important clinical guidance would be "management of red eye in contact lens wearers" and "management of chemical eye injury", which were created with the ophthalmologists. A series of 'red flags' which would warrant referral were also listed and these were accompanied by the specific serious diagnoses that they would need to formally rule out. The ophthalmologists had suggested in the past that they were being called inappropriately by the ED so, although not exhaustive, this list would give staff more confidence to refer when needed.

Given that junior doctors on rotation would likely see relatively few such patients overall and would likely have little need in the future to know extensive amounts of clinical ophthalmology, a pro forma with accompanying online advice would ensure that salient clinical features were elucidated. These could then be interpreted by senior ED or ophthalmology staff if necessary.

Improving patient flow

To streamline patient flow, it was agreed that any referral from an optician or external doctor should be considered for direct ophthalmology referral, unless the problem was readily manageable by the ED. Furthermore, a referral pathway was generated to ensure that two specialist registrars are readily contactable in-hours if advice from the eye clinic nurse is unavailable. There were no clinical circumstances identified in which a triage nurse should directly refer to the ophthalmologists.

\section{Strategy}

Two junior doctors (the authors), an ED consultant, one ophthalmology consultant, and one ophthalmology registrar were at the forefront of devising and implementing these interventions, with help from other staff later on in the process, as detailed below. Following the initial ideas, changes were made based on further testing and constructive feedback.

PDSA Cycle 1: the previous pro forma was double-sided with areas for a full clerking. Our initial pro forma was similar, but we felt that having separate areas for history would make the document too long and a basic eye history did not need to be prescriptive. We therefore simply created a one-sided 'eye examination pro forma' and the history could be written on the casualty card. However, we did include a separate section on the pro forma to remind people to ask about CL use. This concept was agreed upon by senior ED and ophthalmology staff.

PDSA Cycle 2: there was a possibility that this contact lens status part of the pro forma might be ignored and dismissed by users as an unnecessary 'tick box' exercise. An ED consultant suggested that explaining on the pro forma why CL status is important would help to educate users and, once they knew this, it would prompt them to ask about this for each patient. This was supported by preliminary testing by ENPs where all of the 17 pro formas used had this section completed.

PDSA Cycle 3: following this trial, some of the formatting and spacing of the different sections was altered to highlight certain areas. A more detailed explanation of the new follow-up guidance was also included on the pro forma and this linked with the new emergency clinic referral pathways, as follow-up planning was an obvious failing identified in the audit.

The "red eye in a contact lens wearer" and "chemical eye injury" guidelines were drawn up jointly between the ED and ophthalmology seniors and only minor changes were then made based on the specific management for each condition. The 'red flag' list was similarly a joint senior effort but we felt that the diagnosis being queried should be included rather than just a list of clinical findings, again, to aid the ED clinician when referring.

PDSA Cycle 4: the initial plan was that the ophthalmologists carry portable phones to receive referrals. However, when this was trialled for two weeks, they felt it was inappropriate if they were called in the middle of a delicate procedure, for example. They now, therefore, carry bleeps instead, which they do not need to respond to straightaway. Furthermore, the eye clinic nurses are now more experienced so they are still the first point of contact during the day, but registrars can be bleeped if needed.

We also ensured that the pro forma, clinical guidelines, and referral pathways are fully integrated, from initial clerking to appropriate management, specialist referral and follow-up. This, along with the specific changes above, would help to fulfil our aims to improve the overall clinical management of patients presenting with acute red eye(s), streamline their stay in the department - to the benefit of the patients and clinicians - and optimise their post-hospital care.

The staff involved were happy with the interventions and the relevant documents were uploaded on to the intranet, intended to be used for all patients presenting with eye problems. In order to publicise these changes, posters were displayed around the ED for clinicians and some were also directed towards patients, asking them to tell their doctor if they were CL wearers. The ED consultants were specifically made aware of the changes and emails were sent to other ED members. Similarly, given that there 
was continuous senior ophthalmology involvement, it was relatively simple to ensure that their department also subscribed to these changes. It would be difficult to personally drive the project forward as we were both moving hospitals soon after the implementation period but the senior doctors and ENPs involved would still be present and would be able to do this.

We planned to reaudit retrospectively about nine months later to allow time for the new referral pathways and pro forma to become embedded in to daily practice before the commencement of our study period; from our previous data, it would take 4-5 months for one hundred patients to be seen in the ED who would fulfil our study criteria.

\section{Results}

With our new system having been implemented in August 2013, we re-audited the ED medical notes of one hundred consecutive patients (excluding the few whose clerking was recorded electronically) presenting with acute red eyes between October 2013 and March 2014 to assess if there had been an improvement in the aforementioned parameters.

With regards to our primary aim of improving overall patient assessment and management, the new pro forma was used in $28 \%$ of cases and the old pro forma was actually used five times. CL status was documented $54 \%$ of the time, compared to $63 \%$ before our interventions. As before, pro forma use was associated with a much higher rate of $\mathrm{CL}$ status documentation: without a pro forma, this was $40 \%$, whilst with the pro formas, it was $88 \%$ and $83 \%$ (new and old pro formas, respectively).

We also analysed performance based on clinician grade during this audit cycle. ENPs used a pro forma the most and also documented CL status most frequently ( $82 \%$ and $86 \%$ respectively). Interestingly, only one of the six clerkings by ED registrars contained $\mathrm{CL}$ documentation.

The measurement of visual acuity was also recorded less frequently, with $71 \%$ of notes containing formal acuity documentation this cycle compared to $77 \%$ previously. Again, this is correlated with the pro forma. When used, acuity was always documented; when not used, this was only included $40 \%$ of the time. Similarly, pin hole correction was measured appropriately in only $38 \%$ of cases: $73 \%$ of time when the new pro forma was completed and $16 \%$ when no pro forma was used.

The ophthalmologists also suggest that everyone with red eye is examined with a slit lamp and fluorescein stain. These were used $47 \%$ and $34 \%$ of the time respectively, though it is difficult to know whether or not fluorescein has actually been used, as the finding of "no corneal abrasions" can be made with or without staining; both tools were used $42 \%$ of the time initially. The slit lamp was used $89 \%$ of the time by ENPs and $31 \%$ by doctors.

With respect to treatment, a specifically incorrect antibiotic was not prescribed on any occasion when the new pro forma was used. Without a pro forma, there were five instances when an incorrect antibiotic was given to a known contact lens wearer; this is the same number as pre-intervention. Finally, $52 \%$ of patients seen with a new pro forma had a safety net or follow-up documented, whilst this figure was $69 \%$ for those without a pro forma.

In terms of patient flow and appropriate specialist referrals, there were only five instances when a patient identified as having red flag clinical features was not referred, compared to twelve before. Similarly, referrals were mainly made when red flags were present, with only four non-red flag referrals being made, compared to eight before. Clearly, a specialist opinion may be needed even without red flags, but this does suggest that referrals remain appropriate. It is difficult to quantitatively gauge how well the new referral pathways are working. Even trying to demonstrate a decrease in the length of time it now takes to obtain specialist advice would be very crude and uninformative given the various confounding factors involved and, in any case, it would be difficult to measure this retrospectively. Change here is best assessed qualitatively, as the initial aim was to make it generally easier to make a referral so that the whole process is more efficient. The long-standing ENPs and consultants report that the new pathways continue to work well and have become a recognised part of the overall patient flow management of the department, with timely specialist advice nearly always available.

See supplementary file: ds3404.pdf - "Post-intervention data, new pro forma and clinical guidelines"

\section{Lessons and limitations}

Our interventions did not produce the outcomes we had hoped. On reflection, there are a number of lessons we have learned that should help us to succeed in further cycles of this project and in other similar undertakings. Firstly, it is crucial that the stakeholders in this case, the ED doctors and nurses - are aware of the rationale for change. Some clinicians may feel patronised by a pro forma and fixed clinical guidelines, especially as they become more senior, but it is important that they understand that patient safety is being affected and that objective data suggests that these interventions can help. Secondly, the large disparity in our findings in cases when a pro forma was used and the results of our junior doctor survey suggest a need for education amongst clinicians. Similarly, slit lamp and fluorescein examination were often omitted from patient assessments, which could be rectified by targeted teaching. Clinical education and non-technical interventions must therefore be implemented simultaneously to gain the greatest benefit. Thirdly, it is vital that someone can drive the intervention process forward once it is implemented. Establishing this longevity in the short- then medium-term is key. Unfortunately, we both moved hospitals by this time and the consultant involved had larger projects to oversee. We perhaps naively assumed that the changes would persist regardless. Next time, we will need to involve permanent staff in the implementation and education aspects to ensure a continuity of responsibility while junior doctors rotate in and out of the department.

The main limitation of the study was that we had no objective methods to measure the effectiveness of the new referral pathways 


\section{BMJ Quality Improvement Reports}

other than the qualitative survey data. It may also have been useful to formally gauge the opinions of the ED staff regarding the new interventions during the initial implementation phase, especially those that had experienced the old system, to find out any alterations that could have been made to improve their use. This may be something we do before the next audit cycle.

\section{Conclusion}

Introduction of a new pro forma and integrated clinical guidelines had little overall effect, mainly because their use was not universal. Our data were much better when the new systems were used. This vindicates our approach to the problem, but unearths other problems associated with implementing change across a large, busy department, including operational, logistical, and educational considerations.

We have devised various ways of addressing the multiple problems associated with mismanagement of patients who present acutely with red eye(s). We envisage that excellent outcomes can ensue once these are all better integrated within the department. The clinical and Trust-related problems with the non-identification of CL wearers were improved with the introduction of the new pro forma and related clinical guidelines, and greater benefit can be gained once these are used more frequently in the department. This has helped to counteract the relative lack of specific undergraduate acute ophthalmology teaching and the low individual exposure to such patients in the ED, which are major issues that we identified. Unfortunately, we did not adequately deal with the issue that most juniors will not have experience using corneal staining and slit lamp examination; even though these were included on the pro forma as part of the full examination, many simply were not able to perform this. The provision and accessibility of specialist advice has been improved with the new referral pathways. These work well and ensure that there is sufficient back-up if some members of the ophthalmology team are unavailable. The red flag guidelines have also improved the identification of those with worrying clinical features and have helped to make referrals easier and patient flow more efficient.

The next steps in this project are to address these issues and ensure that there is good local backing for the changes, with the knowledge that simple alterations in practice can considerably reduce the risk of life-changing complications. We plan to implement specific junior doctor teaching early in their attachments to facilitate the appropriate use of slit lamps and corneal staining and to improve the prominence and accessibility of the new systems within the department. We will then re-audit in November 2014

The junior doctors we surveyed graduated from a variety of medical schools in the UK which suggests that inadequate undergraduate ophthalmology teaching is commonplace. Similarly, underexposure to these patients whilst working is likely to be a recurring problem in other hospitals. Therefore, whilst other EDs may already have efficient systems in place for obtaining specialist patient reviews, many will still experience the problem of doctors needing further specific guidance where a lack of basic knowledge, regarding $\mathrm{CL}$ use for example, can have disastrous consequences. Therefore, the systems we have described could be introduced within many departments to ensure that these problems are addressed and that patient safety is optimised.

\section{References}

1. http://www.hscic.gov.uk/catalogue/PUB13464. Health and Social Care Information Centre: Accident and Emergency Attendances in England, 2012-2013

2. Local audit carried out in November 2012 at King's College Hospital

3. Willcox MD. Pseudomonas aeruginosa infection and inflammation during contact lens wear: a review. Optometry and vision science : official publication of the American Academy of Optometry. 2007; (4):84

4. http://www.aoa.org/documents/CPG-11.pdf

5. http://cks.nice.org.uk/conjunctivitis-infective\#!topicsummary - NICE Clinical Knowledge Summaries: Conjunctivitis infective

6. Rose, P. (2007) Management strategies for acute infective conjunctivitis in primary care: a systematic review. Expert Opinion on Pharmacotherapy 8(12), 1903-1921.

\section{Declaration of interests}

Nothing to declare

\section{Acknowledgements}

Dr. Graham Fleming for helping to push through these changes with the ophthalmology department and for his ongoing advice,

Dr. Jeff Keep for introducing us to the idea of quality improvement and for his advice early on in the project,

Dr. Anish Patel for his involvement in the project from the ophthalmologists' perspective and for championing the changes within his own department 